\title{
Unusual presentation of patients with myelomatosis in Malawi
}

\author{
RA TOZER, AS CLEAR, DR DAVIES, AND MSR HUTT \\ From the Queen Elizabeth Central Hospital, Blantyre, Malawi, and \\ St Thomas's Hospital Medical School, London SE1 7EH, UK
}

SUMMARY Malignant myeloma is not rare in tropical Africa. Its mode of presentation in Malawi is most often that of a tumour mass in bone. The types of immunoglobulin heavy and light chains in the tumour cells occur with a frequency similar to that observed in temperate countries.

Myelomatosis appears to be more common in North America and Western Europe than in tropical Africa or India, ${ }^{1}$ though it is probable that the condition is underdiagnosed and underrecorded in many tropical countries. In the USA, myelomatosis occurs with equal frequency in black and white Americans. ${ }^{2}$

Most patients with myelomatosis present with evidence of a systemic disease, often with widespread pain, especially in the back, and symptoms of anaemia. ${ }^{3}$ Occasionally, these symptoms are accompanied by more localised bone pain and tenderness or a pathological fracture but it is uncommon for the presentation to be due to the effects of a single tumour mass. In contrast, this presentation appears to be common in Malawian Africans, and in this paper we review our experience since 1969.

\section{Patients and methods}

Since 1969 the Department of Morbid Anatomy at St Thomas's Hospital Medical School has undertaken all the diagnostic histopathology for government and mission hospitals throughout Malawi. After formalin fixation all specimens are sent to the Central Pathology Laboratory in Blantyre, where they are repacked in sealed plastic envelopes and forwarded to London by air once a week.

Paraffin blocks of all cases coded as myeloma or plasmacytoma (SNOP) in the Malawi Histopathology Registry at St Thomas's Hospital Medical School were retrieved, and further sections were cut and stained with haematoxylin and eosin and methyl green pyronin for light microscopy. In all cases in which a histopathological diagnosis of myeloma was confirmed a peroxidase-anti per-

Received for publication 3 December 1979 oxidase (PAP) modification of the immunoenzyme bridge method was used to determine the type of heavy and light chain immunoglobulin produced by the tumour cells. ${ }^{4}$

We also reviewed the records of all patients diagnosed as multiple myelomatosis by standard haematological and biochemical techniques in the Central Laboratory of the Queen Elizabeth Hospital, Blantyre.

\section{Results}

During the period under review, 1969-78, 29 patients were diagnosed as myelomatosis in Malawi (Table 1).

Table 1 Clinical presentation of 29 patients with myelomatosis

\begin{tabular}{|c|c|c|c|}
\hline & $\begin{array}{l}\text { Queen Elizabeth } \\
\text { Central Hospital }\end{array}$ & $\begin{array}{l}\text { Other } \\
\text { hospitals }\end{array}$ & Total \\
\hline $\begin{array}{l}\text { Cases presenting as } \\
\text { a single tumour }\end{array}$ & 9 & 11 & 20 \\
\hline $\begin{array}{l}\text { Cases presenting with } \\
\text { systemic (multiple) } \\
\text { manifestations }\end{array}$ & 4 & 5 & 9 \\
\hline
\end{tabular}

Twenty of these patients presented with a local tumour mass or pathological fracture, and the initial diagnosis was made histologically from a biopsy specimen (Table 1). Nine of these patients were seen in Queen Elizabeth Central Hospital, Blantyre, and 11 in other hospitals throughout Malawi. In the majority of these patients the clinical diagnosis was bone tumour of uncertain type, and myelomatosis had not been suspected clinically at the time of biopsy. The histopathological features in all 20 cases were typical of a myeloma.

During the same period nine patients presented with the classical clinical features of multiple mye- 
lomatosis. The final diagnosis in these cases was based on the results of either marrow aspiration or serum electrophoresis or both.

Twenty patients presented with a localised tumour. There were 15 men and five women and their ages ranged from 13 to 70 years (approximate age), though most were over 40 years. In 19 of the 20 patients the tumour mass was in bone. In four, this had caused a pathological fracture; in the others the patient noticed a lump or had local pain with bone destruction on $x$-ray. Five patients presented with large tumour masses over the ribs or sternum. The clavicle was involved in two cases, humerus in three, tibia in three, femur in two, ilium in one, skull in one, vertebra in one, and in one the bone was not specified.

Unfortunately, it was not possible to do full investigations on most of these patients, but in five there was either evidence of multiple lesions radiologically or they had monoclonal globulins in the serum. Moreover, eight of the 18 patients in whom follow-up data were available died within a few weeks or months of their diagnostic biopsy, and it is likely that most of these had multiple myelomatosis.

Immunoperoxidase studies on the initial tumour are summarised in Table 2 . We judged the tumour to be positive when the cytoplasm of plasma cells contained reaction product. Artifactual staining was seen in many blocks where curettings of a lesion had been taken and cells at the surgical edge were positive with several antisera: this "crush artifact" was disregarded-as was staining of connective tissue and serum in vessels. Only one case was negative for light chains and the three heavy chains: this specimen remained negative after trypsin digestion and also after staining for $\operatorname{IgE}$ and IgD.

Table 2 Characterisation of immunoglobulin chains in tumour cells of 20 cases

\begin{tabular}{lllll}
\hline & Kappa & Lambda & None & Total \\
\hline IgG & 7 & 4 & 0 & 11 \\
IgM & 0 & 0 & 0 & 0 \\
IgA & 3 & 2 & 0 & 5 \\
None & 2 & 1 & 1 & 4 \\
Total & 12 & 7 & 1 & 20 \\
\hline
\end{tabular}

\section{Discussion}

There are few reports on myelomatosis in Africans. Waldbaum and Gelfand ${ }^{5}$ reported 12 cases from Salisbury, Rhodesia seen between 1969 and 1972. Most of their patients were over 50 years and presented with systemic symptoms, but tumour formation was an initial feature in two patients, involving the rib in one and the humerus in another. Other reports on the incidence of leukaemias and lymphomas in Africa indicate the comparative rarity of this tumour ${ }^{6}$ but stress that it is probably underdiagnosed. Wright ${ }^{7}$ refers to 46 cases of plasmacytoma diagnosed in Uganda from 1964 to 1968 and noted that 36 occurred in the skeleton, often as part of multiple myelomatosis. Some of these patients presented with tumour masses, as in the present series from Malawi. Myelomatosis appears to occur as frequently in Jamaican and US blacks as in the white population of North America. ${ }^{3}$ Our experience in Malawi suggests that myelomatosis is probably commoner in Africa than earlier reports suggest. Without the availability of a free histopathological service on a country-wide basis over half of the present cases may not have been diagnosed. The majority of the patients with local bone tumours have been diagnosed since 1974 when the service became more widely used. An unusual feature of myelomatosis in Malawi, though also seen in Uganda, is the frequent clinical presentation with a single and often large tumour mass in bone. Moreover, the distribution of these presenting bone tumours is different from that reported in patients with multiple myelomatosis in other parts of the world. In a series from the USA, the three most common sites of bone involvement in myelomatosis were vertebral column $(66 \%)$, rib $(44 \%)$, and skull $(41 \%){ }^{8}$ This is in marked contrast to this series in which nine of the $20(45 \%)$ tumours were in the long bones of the arm or leg and only one patient presented with a vertebral lesion. However, despite these differences in the clinical presentation and in the site of the bone tumour, the pattern of heavy and light chain production by these tumours is similar to that seen in other parts of the world. ${ }^{9}$

We have reason to believe that only a proportion of patients seen in hospital with a tumour mass will have a biopsy performed, and the histological registry of tumours must underestimate the true incidence of the disease in Malawi. Even so, the likelihood of a biopsy being taken from a readily visible tumour is much greater than the detailed investigation of a patient with an obscure medical condition in a remote rural hospital with few laboratory facilities. During the period under review, 1969-78, a full diagnostic haematology service was available only at the Central Laboratory at Queen Elizabeth Hospital, Blantyre. It is, therefore, probable that cases with the typical multiple form of the disease, presenting without tumour formation, are not diagnosed, especially when the index of clinical suspicion is low and infectious diseases predominate.

Even if typical cases of multiple myelomatosis are 
markedly underdiagnosed, these 18 cases suggest that the tumour behaves differently in Africans in Malawi. The reason for this is not apparent but it is of some interest that chloroma, which is a solid tumour manifestation of myeloid leukaemia, is commoner in Africa than in Europe or the USA, ${ }^{10}$ and Burkitt's lymphoma also affects bones more frequently than other types of lymphoma seen in temperate climates. In none of these diseases can these manifestations be explained solely on the basis of late diagnosis, and it seems probable that some, as yet undefined, host factor may be responsible.

Our thanks are due to the clinicians in many hospitals throughout Malawi who submitted these cases; to the medical laboratory scientific officers at St Thomas's Hospital Medical School for histopathological services; and to Miss B Evans for technical assistance with the immunoperoxidase investigations. The Ministry of Health of Malawi has granted permission to publish.

\section{References}

1 Doll R, Payne P, Waterhouse J. Cancer Incidence in Five Continents. Publication of the International
Union against Cancer. Berlin, New York: Springer, 1966.

${ }^{2}$ Carbone PP, Kellerhouse LE, Gehan EA. Plasmacytic myeloma. Am J Med 1967;42:937-48.

${ }^{3}$ Talerman A. Clinico-pathological study of multiple myeloma in Jamaica. Brit J Cancer 1969;23:285-93.

${ }^{4}$ Sternberger LA. Immunocytochemistry. 2nd ed. New York: John Wiley and Sons Inc, 1979.

${ }^{5}$ Waldbaum B, Gelfand M. Myelomatosis in the Rhodesian African. Trop Geogr Med 1974;26:26-30.

${ }^{6}$ Edington GM, Maclean CMU. A cancer rate survey in Ibadan, Western Nigeria, 1960-63. Brit J Cancer $1965 ; 19: 471-81$.

7 Wright DH. Lymphoreticular neoplasms. Recent Results Cancer Res 1973;41:270-91 (see p. 286).

${ }^{8}$ Spjut HJ, Dorfman HD, Fechner RE, Ackerman LV. Tumors of Bone and Cartilage (Atlas of Tumor Pathology, Second Series, Fascicle 5). 1971. Armed Forces Institute of Pathology, Washington DC, 207.

${ }^{9}$ Malpas JS. Myeloma. Brit J Hosp Med 1977;18: 478-85.

${ }^{10}$ Davies JNP, Owor R. Chloromatous tumours in African children in Uganda. Brit Med $J$ 1965;2: 405-7.

Requests for reprints to: Professor MSR Hutt, Unit of Geographical Pathology, Department of Morbid Anatomy, St Thomas's Medical School, London SE1 7EH, UK. 